\title{
COMPARISON THEOREMS FOR INFINITE SYSTEMS OF DIFFERENTIAL-FUNCTIONAL EQUATIONS AND STRONGLY COUPLED INFINITE SYSTEMS OF FIRST ORDER PARTIAL DIFFERENTIAL EQUATIONS
}

\author{
J. SzARSKI
}

ABSTRACT. The first part of the paper deals with an infinite system of weakly coupled (this means that every equation contains derivatives of only one unknown function) differential-functional equations of the form

$$
u_{x}^{i}(x, y)=f^{i}\left(x, y, u(x, y), u_{y}^{i}(x, y)\right)(i=1,2, \ldots) \text { in } W=\{(x, y):
$$
$\left.x_{0} \leqq x<x+b, y=\left(y_{1}, \ldots, y_{n}\right) \in R^{n}\right\}$. Here $u=\left(u^{1}, u^{2}, \ldots\right)$ : $W \ni(x, y) \rightarrow u(x, y)=\left(u^{1}(x, y), u^{2}(x, y), \ldots\right) \in \ell^{\infty}$ is the unknown function, $u_{y}^{i}(x, y)=\operatorname{grad}_{y} u^{i}(x, y)$ and $f^{i}$ is a functional of the map $u$.

For the problem with the initial conditions

$$
u^{i}\left(a_{i}, y\right)=p^{i}(y) \quad(i=1,2, \ldots)
$$

following questions are discussed: error bounds for an approximate solution, uniqueness of the solution and its continuous dependence on the right hand sides of the system and on the initial functions.

The results of the first part are applied to similar questions concerning a strongly coupled infinite system of first order partial differential equations

$u_{x}^{i}(x, y)=g^{i}\left(x, y, u(x, y), u_{y 1}(x, y), u_{y}^{i}(x, y)\right)(i=1,2, \ldots)$, where $u_{y 1}$ $=\left(u_{y_{1}}^{1}, u_{y_{1}}^{2}, \ldots\right)$. The derivatives $u_{y_{1}}^{j}(j=1,2, \ldots)$ are responsible for the system to be strongly coupled and consequently the functions $u^{j}$ are supposed to belong to a special class of analytic functions with respect to the variable $y_{1}$. This class was first taken advantage of by $\mathrm{K}$. Nickel [3] in the theory of strongly coupled parabolic systems of nonlinear second order differential equations. The analyticity of $u^{j}$ with respect to $y_{1}$ is essential in questions treated in this paper. This is shown by a counter-example constructed by A. Plis [5] in which for a strongly coupled system of two linear equations there is no uniqueness for the Cauchy problem in the class $C^{\infty}$. The local uniqueness (and also existence) of the solution, which is analytic with respect to $y$ and belongs to the class $C^{1}$ with respect to $x$, was proved by M. Nagumo [2] in the finite case of differential equations, but under more restrictive assumptions on analyticity of the right hand sides of the strongly coupled system with respect to all arguments but the variable $x$. The argument of the first part is similar to that used by A. Plis for a finite and weakly coupled system of first order partial differential equations in [4] and by the author in [7] and [8], where an existence theorem for infinite systems of differential-functional equations is proved too.

Received by the editors on April 13, 1978. 
1. Preliminary remarks and definitions. Put

$$
W=\left\{(x, y): x_{0} \leqq x<x_{0}+b, y \in R^{n}\right\},
$$

where $0<b<\infty$, let $V_{0}$ be a subset (possibly empty) of $\left\{(x, y): x<x_{0}\right\}$ and denote $W_{0}=W \cup V_{0}$. Let $C_{\infty}\left(W_{0}\right)$ stand for the real Banach space of mappings

$$
\begin{aligned}
w & =\left(w^{1}, w^{2}, \ldots\right): W_{0} \ni(x, y) \rightarrow w(x, y) \\
& =\left(w^{1}(x, y), w^{2}(x, y), \ldots\right) \in \ell^{\infty},
\end{aligned}
$$

where $w^{j}$ are continuous in $W$, with the finite norm

$$
\|w\|=\sup \left\{\left|w^{j}(x, y)\right|:(x, y) \in W_{0}, j=1,2, \ldots\right\} .
$$

We recall that $\iota^{\infty}$ is the real Banach space of bounded sequences $s=$ $\left(s^{1}, s^{2}, \ldots\right)$ with the finite norm

$$
\left.\|s\|_{r^{\infty}}=\sup \left\{\left|s^{j}\right|: j=1,2, \ldots\right)\right\} .
$$

For $w \in C_{\infty}\left(W_{0}\right)$ and for a fixed $x \leqq x_{0}$ we put

$$
\|w\|_{x}=\sup \left\{\left|w^{j}(\tilde{x}, \tilde{y})\right|:(\tilde{x}, \tilde{y}) \in W_{0}, \tilde{x} \leqq x, j=1,2, \ldots\right\} .
$$

We obviously have

$$
\|w\|_{x}=\|w\| \text { for } x \geqq x_{0}+b .
$$

If for $w \in C_{\infty}\left(W_{0}\right)$ we have $w^{j} \in C^{1}(W)(j=1,2, \ldots)$, then we write $w \in C_{\infty}^{1}(W)$.

A function

$$
\varphi: R \rightarrow R
$$

is said to be of class $\mathfrak{B}(K)$, where $K$ is a positive constant, if the functions $\varphi^{(j)}(j=0,1, \ldots)$ are continuous in $R$ and

$$
\begin{aligned}
\|\varphi\| & =\sup \{|\varphi(t)|: t \in R\}<\infty,\left\|\varphi^{(/)}\right\| \\
& =\sup \left\{\left|\varphi^{(\iota)}(t)\right|: t \in R\right\} \leqq K^{(\iota)}(\ell=1,2, \ldots) .
\end{aligned}
$$

The following theorem is due to S. N. Bernstein [1].

THEOREM (OF BERNSTEIN). If $\varphi_{1}, \varphi_{2} \in \mathfrak{B}(K)$, then

$$
\left\|\varphi_{1}^{\prime}-\varphi_{2}^{\prime}\right\| \leqq K\left\|\varphi_{1}-\varphi_{2}\right\| .
$$

A function

$$
\left(\psi^{1}, \psi^{2}, \ldots\right)=\phi: W_{0} \rightarrow \iota^{\infty}
$$

will be said to be of class $\mathfrak{B}\left(K, y_{1}, W\right)$ if for any fixed values $x, y_{2}, \ldots, y_{n}$, 
such that $x_{0} \leqq x<x_{0}+b$, and for every $j=1,2, \ldots, \psi^{j}$ belongs to the class $\mathfrak{B}(K)$ as function of the variable $y_{1}$.

2. Comparison theorems for an infinite system of weakly coupled differential-functional equations.

THEOREM 1. Suppose the real functions $f^{i}(x, y, s, w, q)(i=1,2, \ldots)$ are defined for $(x, y) \in W, s \in \iota^{\infty}, w \in Y, q=\left(q_{1}, \ldots, q_{n}\right) \in R^{n}$, where $Y$ is a subset (not necessarily a linear subspace) of $C_{\infty}\left(W_{0}\right)$. Assume the Lipschitz condition

$$
\begin{aligned}
& \left|f^{i}(x, y, s, w, q)-f^{i}(x, y, \tilde{s}, \tilde{w}, \tilde{q})\right| \\
& \quad \leqq B\left(\|s-\tilde{s}\|_{\iota^{\infty}}+\|w-\tilde{w}\|_{x}\right)+C \sum_{k=1}^{n}\left|q_{k}-\tilde{q}_{k}\right|(i=1,2, \ldots)
\end{aligned}
$$

to be satisfied with some constants $B>0$ and $C \geqq 0$. Let the constants $a_{i}$ satisfy the inequalities

$$
x_{0} \leqq a_{i}<x_{0}+h<x_{0}+\min \left\{b,(2 B)^{-1}\right\} .
$$

Let $u \in Y \cap C_{\infty}^{1}(W)$ and $v \in Y \cap C_{\infty}^{1}(W)$ be solutions of the following equations and inequalities

$$
\begin{aligned}
& u_{x}^{i}(x, y)=f^{i}\left(x, y, u(x, y), u, u_{y}^{i}(x, y)\right)(i=1,2, \ldots),(x, y) \in W, \\
& \left|v_{x}^{i}(x, y)-f^{i}\left(x, y, v(x, y), v, v_{y}^{i}(x, y)\right)\right| \leqq \varepsilon,(i=1,2, \ldots) \\
& (x, y) \in W \\
& \left|v^{i}\left(a_{i}, y\right)-u^{i}\left(a_{i}, y\right)\right| \leqq \varepsilon, \quad\left|v^{i}(x, y)-u^{i}(x, y)\right| \leqq \varepsilon,(i=1,2, \ldots) \\
& (x, y) \in V_{0} .
\end{aligned}
$$

where $u_{y}^{i}(x, y)=\operatorname{grad}_{y} u^{i}(x, y)$, and $\varepsilon \geqq 0$ is a given constant.

Under these assumptions we have the following error estimate

$$
\|u-v\| \leqq \varepsilon A^{p},
$$

where $p=[b / h]+1, A=(1+h)(1-2 h B)^{-1}$.

The fact that in (2.1) we have $\|w-\tilde{w}\|_{x}$ instead of $\|w-\tilde{w}\|$ means that for two functions $w, \tilde{w} \in Y$, such that $w(\tilde{x}, y)=\tilde{w}(\tilde{x}, y)$ for $\tilde{x} \leqq x, f^{i}$ as functional of $w$ ( $x$ and other arguments being fixed) takes the same values; in other words, $f^{i}$ is a functional of Volterra type. In [7] a special case of Volterra functionals was considered. The possibility of introducing general Volterra functionals was pointed to by A. Pelczar.

Proof. We will prove first that

$$
\|u-v\|_{x_{0}+h} \leqq \varepsilon A .
$$


Put $z=u-v$ and notice that by (2.1), (2.3) and (2.4) we have for

$$
\begin{aligned}
& (x, y) \in W^{h}=W \cap\left\{(x, y): x \leqq x_{0}+h\right\} \\
& \left|z_{x}^{i}(x, y)\right|=\left|f^{i}\left(x, y, u(x, y), u, u_{y}^{i}(x, y)\right)-v_{x}^{i}(x, y)\right| \\
& \leqq\left|f^{i}\left(x, y, u(x, y), u, u_{y}^{i}(x, y)\right)-f^{i}\left(x, y, v(x, y), v, v_{y}^{i}(x, y)\right)\right| \\
& +\left|f^{i}\left(x, y, v(x, y), v, v_{y}^{i}(x, y)\right)-v_{x}^{i}(x, y)\right| \\
& \leqq B\left(\|z(x, y)\|_{\infty}+\|z\|_{x_{0}+h}\right)+C \sum_{k=1}^{n}\left|z_{y k}^{i}(x, y)\right|+\varepsilon .
\end{aligned}
$$

Hence, it follows that in $W^{h}$ and for every fixed $i=1,2, \ldots$ we get

$$
\left|z_{x}^{i}(x, y)\right| \leqq 2 B\|z\|_{x_{0}+h}+C \sum_{k=1}^{n}\left|z_{y k}^{i}(x, y)\right|+\varepsilon .
$$

On the other hand, inequalities (2.5) imply that

$$
\left|z^{i}\left(a_{i}, y\right)\right| \leqq \varepsilon .
$$

By the differential and initial inequalities (2.8) and (2.9) we conclude [6] that for every $i=1,2, \ldots$ we have in $W^{h}$

$$
\left|z^{i}(x, y)\right| \leqq\left(2 B\|z\|_{x_{0}+h}+\varepsilon\right)\left|x-a_{i}\right|+\varepsilon
$$

and consequently, since $\left|x-a_{i}\right| \leqq h$ in $W^{h}$ and, by

$$
\left|z^{i}(x, y)\right| \leqq \varepsilon \text { for }(x, y) \in V_{0},
$$

we get

$$
\|z\|_{x_{0}+h} \leqq\left(2 B\|z\|_{x_{0}+h}+\varepsilon\right) h+\varepsilon .
$$

Since $2 h B<1$ by (2.2), it follows from the last inequality that

$$
\|z\|_{x_{0}+h} \leqq \varepsilon A,
$$

which is equivalent to (2.7).

Now, substitute $x_{0}+h$ for $x_{0}, \tilde{W}=W \backslash W^{h}$ for $W, W^{h} \cup V_{0}$ for $V_{0}, \varepsilon A$ for $\varepsilon$ and $x_{0}+h$ for $a_{i}(i=1,2, \ldots)$ and check that by (2.7) we have

$$
\left|v^{i}\left(x_{0}+h, y\right)-u^{i}\left(x_{0}+h, y\right)\right| \leqq \varepsilon A,\left|v^{i}(x, y)-u^{i}(x, y)\right| \leqq \varepsilon A
$$

for $(x, y) \in W^{h} \cup V_{0}$.

Since $\varepsilon \leqq \varepsilon A$ and (2.4) is satisfied in $\tilde{W}$, we see that the previous argument holds in $\tilde{W}$ and we get

$$
\|u-v\|_{x_{0}+2 h} \leqq \varepsilon A^{2} .
$$

Proceeding thus step by step ( $p$ times) and observing that $p h>b$ and consequently $x_{0}+p h>x_{0}+b$, we finally get (2.6). 
From Theorem 1 we obtain immediately the following uniqueness criterion.

Theorem 2. Assume that $f^{i}$, defined as in Theorem 1, satisfy the Lipschitz condition (2.1) and that the inequalities (2.2) hold true. Suppose that the initial functions

$$
h^{i}: R^{n} \rightarrow R, \ell^{i}: V_{0} \rightarrow R,(i=1,2, \ldots)
$$

are given.

Under these assumptions the initial value problem for the system (2.3) with the initial conditions

$$
u^{i}\left(a_{j}, y\right)=h^{i}(y), u^{i}(x, y)=\ell^{i}(x, y),(x, y) \in V_{0}(i=1,2, \ldots)
$$

admits at most one solution $u \in Y \cap C_{\infty}^{1}(W)$.

In the uniqueness theorem proved in [8] $B$ was assumed to be sufficiently small.

Proof. For two solutions $u$ and $v$ all assumptions of Theorem 1 are satisfied with $\varepsilon=0$. Hence, by (2.6) we get $u=v$ in $W_{0}$.

In a similar way we deduce the following theorem concerning the continuous dependence of the solution on the right hand sides of the system and on the initial functions.

THEOREM 3. Under the assumptions of Theorem 2 let $u \in Y \cap C_{\infty}^{1}(W)$ be the solution of the problem (2.3), (2.10). For every $\nu=1,2, \ldots$ assume $u^{\nu} \in Y \cap C_{\infty}^{1}(W)$ to be a solution of the system

$$
u_{x}^{\nu i}(x, y)=f^{\nu i}\left(x, y, u^{\nu}(x, y), u^{\nu}, u_{y}^{\nu i}(x, y)\right),(i=1,2, \ldots)(x, y) \in W
$$

and denote

$$
\begin{aligned}
\eta_{\nu}^{i}= & \sup \left\{\mid f^{\nu i}\left(x, y, u^{\nu}(x, y), u^{\nu}, u_{y}^{\nu i}(x, y)\right)\right. \\
& \left.-f^{i}\left(x, y, u^{\nu}(x, y), u^{\nu}, u_{y}^{\nu i}(x, y)\right) \mid:(x, y) \in W\right\}, \\
\mu_{\nu}^{i}= & \sup \left\{\left|u^{\nu i}\left(a_{i}, y\right)-h^{i}(y)\right|: y \in R^{n}\right\}, \\
\lambda_{\nu}^{i}= & \sup \left\{\left|u^{\nu i}(x, y)-\ell^{i}(x, y)\right|:(x, y) \in V_{0}\right\} . \\
\varepsilon_{\nu}= & \sup \left\{\eta_{\nu}^{i}, \mu_{v}^{i}, \lambda_{\nu}^{i}: i=1,2, \ldots\right\},
\end{aligned}
$$

Suppose that

$$
\lim _{\nu \rightarrow \infty} \varepsilon_{\nu}=0 .
$$

Under these assumptions we have

$$
\lim _{\nu \rightarrow \infty}\left\|u-u^{\nu}\right\|=0 .
$$


Proof. The index $\nu$ being fixed for $u$ and $u^{\nu}$, all assumptions of Theorem 1 are satisfied with $\varepsilon=\varepsilon_{\nu}$. Hence, by (2.6) we get

$$
\left\|u-u^{\nu}\right\| \leqq \varepsilon_{\nu} A^{p}
$$

and (2.12) follows by (2.11).

3. Comparison theorems for an infinite strongly coupled system of first order partial differential equations.

THEOREM 4. Let the real functions $g^{i}(x, y, s, r, q)(i=1,2, \ldots)$ be defined for $(x, y) \in W, s \in \ell^{\infty}, r \in \ell^{\infty}, q \in R^{n}$ and assume the Lipschitz condition

$$
\begin{aligned}
& \left|g^{i}(x, y, s, r, q)-g^{i}(x, y, \tilde{s}, \tilde{r}, \tilde{q})\right| \leqq \tilde{B}\left(\|s-\tilde{s}\|_{\infty^{\infty}}+\right. \\
& \left.\|r-\tilde{r}\|_{, \infty}\right)+C \sum_{k=1}^{n}\left|q_{k}-\tilde{q}_{k}\right|
\end{aligned}
$$

to hold true. Let a positive constant $K$ be given and suppose that the constants $a_{i}$ satisfy the following inequalities

$$
\begin{aligned}
& x_{0} \leqq a_{i}<x_{0}+\tilde{h}<x_{0}+\min \left\{b,(2 B)^{-1},(2 B K)^{-1}\right\} \\
& (i=1,2, \ldots) .
\end{aligned}
$$

Suppose $u, v \in C_{\infty}\left(W_{0}\right) \cap C_{\infty}^{1}(W) \cap \mathfrak{B}\left(K, y_{1}, W\right)$, are solutions of the following infinite strongly coupled system of first order partial differential equations and inequalities

$$
\begin{aligned}
& u_{x}^{i}(x, y)=g^{i}\left(x, y, u(x, y), u_{y_{1}}(x, y), u_{y}^{i}(x, y)\right),(i=1,2, \ldots) \\
& (x, y) \in W, \text { where } u_{y_{1}}=\left(u_{y_{1}}^{1}, u_{y_{1}}^{2}, \ldots\right), \\
& \quad\left|v_{x}^{i}(x, y)-g^{i}\left(x, y, v(x, y), v_{y_{1}}(x, y), v_{y}^{i}(x, y)\right)\right| \leqq \varepsilon \\
& \quad(i=1,2, \ldots)(x, y) \in W, \\
& \mid\left(v^{i}\left(a_{i}, y\right)-u^{i}\left(a_{i}, y\right)|\leqq \varepsilon,| v^{i}(x, y)-u^{i}(x, y) \mid \leqq \varepsilon\right. \\
& \text { for }(x, y) \in V_{0},
\end{aligned}
$$

where $\varepsilon \geqq 0$ is a given constant.

Under these assumptions we have the following error estimate

$$
\|u-v\| \leqq \varepsilon \tilde{A}^{\tilde{p}},
$$

where

$$
\tilde{p}=[b / \tilde{h}]+1, \tilde{A}=(1+\tilde{h})[1-2 \tilde{h} \max (\tilde{B}, \tilde{B} K)]^{-1} .
$$

Proof. If we put

$$
Y=C_{\infty}\left(W_{0}\right) \cap C_{\infty}^{1}(W) \cap \mathfrak{B}\left(K, y_{1}, W\right),
$$

then by Bernstein's theorem we have 


$$
w, \tilde{w} \in Y \Rightarrow\left|w_{y_{1}}^{j}(x, y)-\tilde{w}_{y_{1}}^{j}(x, y)\right| \leqq K\|w-\tilde{w}\|_{x}(j=1,2, \ldots)
$$

for $(x, y) \in W$.

Define now

$$
f^{i}(x, y, s, w, q)=g^{i}\left(x, y, s, w_{y_{1}}(x, y), q\right)(i=1,2, \ldots)
$$

for $(x, y) \in W, s \in \ell^{\infty}, w \in Y, q \in R^{n}$; then, by (3.1), (3.2) and (3.8) we see that assumptions (2.1) and (2.2) of Theorem 1 are satisfied with $h=\tilde{h}$ and $B=\max (\tilde{B}, \tilde{B} K)$. Now, notice that solutions $u$ and $v$, belonging to $C_{\infty}\left(W_{0}\right) \cap C_{\infty}^{1}(W) \cap \mathfrak{B}\left(K, y_{1}, W\right)$, of (3.3) or respectively (3.4), (3.5) are solutions of (2.3) or respectively (2.4), (2.5) with $f^{i}$ defined by (3.9) and have properties required in Theorem 1. Hence, the estimate (3.6) follows from (2.6).

As in section 2 Theorem 4 implies the following uniqueness criterion.

THeORem 5. Assume that $g^{i}$, defined as in Theorem 4, satisfy the Lipschitz conditions (3.1) and that the inequalities (3.2) hold. Suppose the initial functions $h^{i}(y)$ and $\iota^{i}(x, y)$ to be given.

Under these assumptions the initial value problem for the infinite strongly coupled system of first order partial differential equations (3.3) with the initial conditions (2.10) admist at most one solution $u \in C_{\infty}\left(W_{0}\right) \cap C_{\infty}^{1}(W)$ $\cap \mathfrak{B}\left(K, y_{1}, W\right)$.

In a similar way we get the following theorem concerning the continuous dependence of the solution on the right hand sides of the strongly coupled system (3.3) and on the initial functions.

THEOREM 6. Under the assumptions of Theorem 5 let $u \in C_{\infty}\left(W_{0}\right) \cap$ $C_{\infty}^{1}(W) \cap \mathfrak{B}\left(K, y_{1}, W\right)$ be the solution of the problem (3.3), (2.10). For every $\nu=1,2, \ldots$ assume $u^{\nu} \in C_{\infty}\left(W_{0}\right) \cap C_{\infty}^{1}(W) \cap \mathfrak{B}\left(K, y_{1}, W\right)$ to be a solution of the system

$$
u_{x}^{\nu i}(x, y)=g^{\nu i}\left(x, y, u^{\nu}(x, y), u_{y_{1}}^{\nu}(x, y), u_{y}^{\nu i}(x, y)\right),(i=1,2, \ldots)(x, y) \in W
$$

and denote

$$
\begin{aligned}
\eta= & \sup \left\{\mid g^{\nu i}\left(x, y, u^{\nu}(x, y), u_{y_{1}}^{\nu}(x, y), u_{y}^{\nu i}(x, y)\right)\right. \\
& \left.-g^{i}\left(x, y, u^{\nu}(x, y), u_{y_{1}}^{\nu}(x, y), u_{y}^{\nu i}(x, y)\right) \mid:(x, y) \in W\right\}, \\
\mu_{\nu}^{i}= & \sup \left\{\left|u^{\nu i}\left(a_{i}, y\right)-h^{i}(y)\right|: y \in R^{n}\right\}, \\
\lambda_{\nu}^{i}= & \sup \left\{\left|u^{\nu i}(x, y)-\iota^{i}(x, y)\right|:(x, y) \in V_{0}\right\}, \\
\varepsilon_{\nu}= & \sup \left\{\eta_{\nu}^{i}, \mu_{\nu}^{i}, \lambda_{\nu}^{i}: i=1,2, \ldots\right\} .
\end{aligned}
$$

Suppose that (2.11) holds.

Under these assumptions we have (2.12).

REMARK. It is obvious that in theorems of section 3 (adequately re- 
formulated) the derivatives of unknown functions with respect to several variables $y$ and of higher order may also be responsible for the system to be strongly coupled.

It is not difficult to see that combining results of section 2 and 3 theorems on strongly coupled systems of differential-functional equations are easy to deduce.

It is clear too that the results of the paper apply in particular to finite systems.

The question of existence of a solution to problem (3.3), (2.10) with properties required in the uniqueness Theorem 5 will be discussed in a subsequent note.

\section{REFERENCES}

1. S.N. Bernstein, Sur une propriété des fonctions entières, Comptes Rendus 176(1923), 1603-1605.

2. M. Nagumo, Über das Anfangswertproblem partieller Differentialgleichungen, Jap. Journal of Math. 18 (1941-43), 41-47.

3. K. Nickel, Fehlerschranken und Eindeutigkeitsaussagen für die Lösungen nichtlinearer, stark gekoppelter, parabolischer Differentialgleichungsysteme, Math. Z. 152 (1976), 33-45.

4. A. Pliś, Generalization of the Cauchy problem for a system of partial differential equations, Bull. Acad. Polon. Sci. 4 (1956), 741-744.

5. — Non-uniqueness in Cauchy's problem for differential equations of elliptic type, Journal of Math. and Mech. 9 (1960), 557-562.

6. J. Szarski, Differential Inequalities, Polish Scientific Publishers, Warszawa 1967.

7. - Generalized Cauchy problem for differential-functional equations with first order partial derivatives, Bull. Acad. Polon. Sci. 24 (1976), 575-580.

8. - Cauchy problem for an infinite system of differential-functional equations with first order partial derivatives, Commentationes Mathematicae 21 (1978), 293-300.

INSTYTUt MATEMATYki UNIWERSYTETU JAGIELloŃSKIEGo, KRaKów.

Institute of Mathematics of the Jagellonian University, KRaków, Poland. 